\title{
Carboxyterminal propeptide of type I procollagen in ELF: elevation in asbestosis, but not in pleural plaque disease
}

\author{
L. Lammi*, L. Ryhänen**,, E. Lakari**, J. Risteli ${ }^{\S}$, P. Pääkkö\# ${ }^{\#}$ E-M. Ruotsalainen**, \\ S. Lähde ${ }^{+}$, V.L. Kinnula**
}

Carboxyterminal propeptide of type I procollagen in ELF: elevation in asbestosis, but not in pleural plaque disease. L. Lammi, L. Ryhänen, E. Lakari, J. Risteli, P. Pääkkö, E-M. Ruotsalainen, S. Lähde, V.L. Kinnula. (C)ERS Journals Ltd 1999.

ABSTRACT: Markers of collagen metabolism may possibly be used in the assessment of pulmonary involvement in asbestosis-related pulmonary diseases. In this study the levels of the carboxyterminal propeptide of type I procollagen (PICP) and the aminoterminal propeptide of type III procollagen (PIIINP) were evaluated in bronchoalveolar lavage fluid (BALF), epithelial lining fluid (ELF) and serum from patients with asbestos related pulmonary and pleural involvement.

Forty-two consecutive patients with occupational exposure to asbestos fibres, who underwent bronchoscopy and bronchoalveolar lavage (BAL) at the time of the diagnosis were investigated. Five patients were diagnosed as having asbestosis, while 37 showed no parenchymal involvement. Of the latter group, 25 had pleural plaques, while 12 had no detectable changes in chest radiographs. The patients were followedup for an average of 7 yrs.

The PICP in BALF and ELF was detectable in all patients with asbestosis and in 8/ 37 subjects without parenchymal involvement. The levels of PICP in BALF and ELF were significantly higher in the asbestosis group compared to the patients without asbestosis $\left(9.8 \pm 1.8 \mu \mathrm{g} \cdot \mathrm{L}^{-1}\right.$ versus $0.6 \pm 1.3 \mu \mathrm{g} \cdot \mathrm{L}^{-1}, \mathrm{p}<0.001$ and $488.9 \pm 208.8 \mu \mathrm{g} \cdot \mathrm{L}^{-1}$ versus $22.6 \pm 50.6 \mu \mathrm{g} \cdot \mathrm{L}^{-1}, \mathbf{p}<0.001$, respectively). Only 1 patient with asbestosis and 3 patients without parenchymal involvement had detectable levels of PIIINP in BALF. The serum levels of PICP and PIIINP did not differ between the patients with asbestosis and those with exposure to asbestos fibres without asbestosis and were within the normal range. None of the 37 patients exposed to asbestos fibres without parenchymal involvement at the baseline developed asbestosis during the follow-up period of $7 \mathrm{yrs}$.

In conclusion, the data show that the carboxyterminal propeptide of procollagen type I, but not the aminoterminal propeptide of type III procollagen is highly elevated in bronchoalveolar lavage fluid and epithelial lining fluid in patients with asbestosis, but not in those without parenchymal involvement. This suggests that the determination of carboxyterminal propeptide of procollagen type $I$ in bronchoalveolar lavage fluid could be used as a marker of parenchymal involvement in patients exposed to asbestos fibres.

Eur Respir J 1999; 14: 560-564.
*Dept of Pulmonary Medicine, Vaasa Central Hospital, Vaasa, Finland. **Dept of Internal Medicine, "Medical Biochemistry, ${ }^{\S}$ Clinical Chemistry, "Pathology, and Diagnostic Radiology, University of Oulu, Oulu, Finland.

Correspondence: V.L. Kinnula

Dept of Internal Medicine

University of Oulu

Kajaanintie $50 \mathrm{~A}$

FIN-90220 Oulu

Finland

Fax: 35883155423

Keywords: Asbestosis asbestos exposure

bronchoalveolar lavage epithelial lining fluid procollagen

Received: January 131999

Accepted after revision April 201999

This work was partly supported by the Finnish Anti Tuberculous Association Foundation, Finland, and the Technology Development Centre, Finland (TEKES).
Exposure to asbestos fibres may lead to hyaline plaques of parietal pleura, asbestos pleurisy, asbestosis and malignant diseases of the lower respiratory tract and pleura. Pleural plaques and asbestosis are the most common nonmalignant pulmonary diseases related to asbestos exposure. The prognosis of asbestosis is variable, but asbestosis usually leads to slowly progressive pulmonary fibrosis.

The various stages in the development of pulmonary fibrosis include inflammation which is initially followed by deposition of type III collagen and later by type I collagen fibres [1]. These collagens are synthesized as procollagen precursor molecules, and propeptides of these procollagens are cleaved in stoichiometric amounts during the collagen formation process [2]. The aminoterminal propeptide of type III procollagen (PIIINP) has been investigated in both the serum and the bronchoalveolar lavage fluid (BALF) of patients with fibrosing alveolitis $[3-5]$ and sarcoidosis $[6,7]$, and previous results suggest that PIIINP could be used in the assessment of these diseases. The carboxyterminal propeptide of type I procollagen (PICP) is theoretically a better marker of collagen synthesis than PIIINP [8]. However, very little is known about PICP in parenchymal lung diseases. Previous results suggest that BALF-PICP reflects the fibrotic process in the lungs, whereas the serum levels of this propeptide play no role in the assessment of these diseases $[5,7]$

No studies are available on the concentration of PICP in BALF, epithelial lining fluid (ELF) or serum in asbestosexposed individuals. Furthermore, very few studies have been conducted on PIIINP following work-related asbestos exposure. Experimental studies with asbestos-exposed rats have histologically shown an increase of type III collagen during the 2 weeks of exposure, followed by a higher proportion of type I collagen [9]. An incease of type III collagen in histological investigations and an increase 
of PIIINP in BALF after 2 months of chrysotile exposure has been shown in sheep [10]. Furthermore, the sheep that had abnormal chest radiograph findings after 18 months exposure to chrysotile had significantly elevated PIIINP in BALF [11]. These experimental exposures have, however, used high and toxic concentrations of asbestos fibres. In humans, the PIIINP was significantly elevated in BALF from subjects with asbestosis and asbestos-associated alveolitis compared with asbestos-exposed workers without disease [12]. Another study on serum PIIINP levels in workers exposed to asbestos fibres suggests that serum PIIINP might be a useful index for the early diagnosis of asbestos-induced pulmonary fibrosis [13]. It has previously been observed that the intensity and duration of asbestos exposure correlates with the quantity of fibres remaining in the lung tissue and asbestos bodies in BALF [14].

The aim of this study was to evaluate the role of the serum, BALF and ELF levels of PIIINP and PICP as markers of pulmonary fibrosis in asbestos-exposed individuals. To further investigate the role of these collagen markers, the patients were followed-up prospectively for an average of 7 yrs.

\section{Materials and methods}

\section{Study population}

The study series included 42 consecutive patients who had been referred to the Pulmonary Department of Päivärinne Hospital (Muhos, Finland) between February 1990 and August 1992 due to a history of occupational asbestos exposure and changes in chest radiographs. The radiological abnormalities included either suspected pleural plaques or an interstitial basal reticular-linear pattern suggestive of fibrosis and asbestosis. The patients underwent bronchoscopy and bronchoalveolar lavage (BAL). Twenty-five patients had pleural plaques, and the chest radiograph was afterwards considered normal in 12 patients. Asbestosis was diagnosed in 5 patients. The diagnosis of asbestosis in 1 patient was based on an open lung biopsy. The remaining four patients had typical changes in chest radiographs, spirometry and diffusion capacity findings and a significant but variable number of asbestos bodies $(\mathrm{AB})$ in BALF, (which were $2.4,12.4,130$, and $772 \mathrm{AB} \cdot \mathrm{mL}^{-1}$ ). Other pulmonary diseases were excluded. The characteristics of the study population are shown in table 1 . The mean follow-up time was 7 yrs (from 5 yrs 7 months to 7 yrs 10 months)

Informed consent was obtained from each subject beforehand, and the Ethics Committee of Päivärinne Hospital approved the research protocol.

\section{Methods}

The chest radiographs included posteroanterior and lateral views. The radiographs were first interpreted by a specialist in pulmonary medicine, and later retrospectively by an experienced thoracic radiologist. Pulmonary function tests, including forced expiratory volume in one second (FEV1) and forced vital capacity (FVC), were made with a flow volume spirometer. Carbon monoxide diffusion capacity of the lung (DL,CO) and $D \mathrm{~L}, \mathrm{CO}$, divided by the alveolar volume $(D \mathrm{~L}, \mathrm{CO} / V \mathrm{~A}$ the specific diffusion coefficient) were analysed using the single-breath technique.
Table 1. - Characteristics of the study population

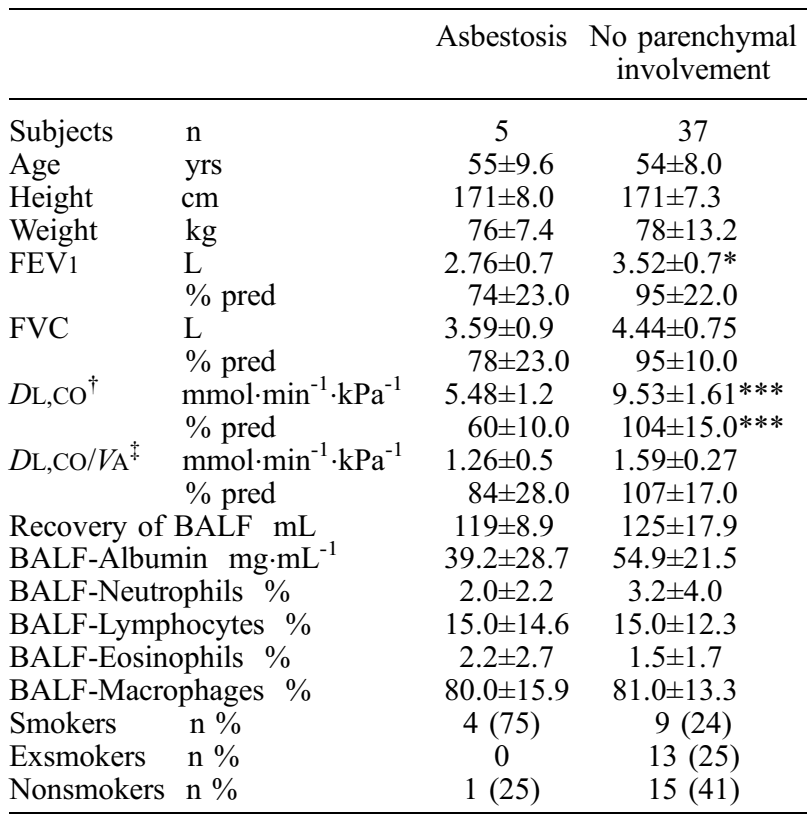

Values are mean \pm SD. All subjects were male. The significance of the difference was tested with the Mann-Whitney U-test. *: $\mathrm{p}<0.05, * * *: \mathrm{p}<0.001 ;{ }^{\dagger}$ : available from 33 patients without parenchymal involvement; ${ }^{*}$ : available from 31 patients without parenchymal involvement. FEV1: forced expiratory volume in one second; FVC: forced vital capacity; DL,CO: carbon monoxide diffusing capacity of the lung; $D \mathrm{~L}, \mathrm{CO} / V \mathrm{~A}$ : carbon monoxide diffusing capacity of the lung, divided by, alveolar volume specific diffusion coefficient; BALF: bronchoalveolar lavage fluid; $\%$ pred: percentage of predicted value.

Fibreoptic bronchoscopy was performed under local anaesthesia with lignocain after premedication with oral diazepam and subcutaneous atropine. A bronchofibreoscope was wedged into the right middle lobe bronchus or into the left lingula. Ten aliquots of $20 \mathrm{~mL}$ saline solution were installed. The recovery of the lavage fluid is shown in table 1 . After centrifugation $(400 \times g$ for $15 \mathrm{~min})$, the cellfree supernatant was stored at $-20^{\circ} \mathrm{C}$ for the PIIINP and PICP assays.

The number of $\mathrm{AB}$ in BALF were determined from Millipore filter and cytocentrifuge (Cytospin 2; Shandon Instruments, Astmoor, UK) preparations. Two or three filter preparations containing $5 \mathrm{~mL}$ of lavage fluid each, and four or five cytocentrifuge preparations containing 0.4 $\mathrm{mL}$ of lavage fluid each were prepared as described previously [7]. Both the Millipore filter and the cytocentrifuge preparations were stained with Pearl's iron. The filter preparations were mounted on glass slides, and both the filter and the cytocentrifuge preparations were covered with coverslips. AB were identified on the basis of the previously described criteria [15], using a light microscope with a $20 \times$ objective lense. The number of $A B$ was expressed as per millilitre of BALF.

Albumin, urea and alanine aminotransferase (ALAT) were measured by standard laboratory methods. Serum levels (S) of PICP and PIIINP were determined by commercial radioimmunoassay kits (Orion; Diagnostica Oy, Oulunsalo, Finland) [16, 17], using human antigens and specific polyclonal antibodies. The radioimmunoassays of BALF-PICP and -PIIINP were conducted as described 
previously [7]. The results were expressed as micrograms of propeptide per litre of BALF or ELF estimated by the urea method [18]. Cell differentials were determined from May-Grünwald Giemsa stained cytocentrifuge preparations.

\section{Statistical analysis}

Non-parametric tests were used in statistical evaluation. The correlations were assessed using the Spearman correlation coefficient test, and the Mann-Whitney U-test was used to assess the differences between the groups. $p$-values $\leq 0.05$ were considered to be significant.

\section{Results}

The lung function parameters and other characteristics of the study population are shown in table 1 . FEV1 and $D$ L,CO were significantly decreased in the patients with asbestosis.

The levels of PICP and PIIINP were analysed in serum, BALF and ELF. All of the 5 patients with asbestosis had significantly higher levels of PICP in BALF and ELF than the 37 patients without parenchymal involvement (table 2 and fig. 1). In the latter group, $6 / 25$ patients with pleural plaques and 2/12 patients without pleural involvement had detectable PICP in BALF and ELF. The levels of the procollagen markers of these two groups did not differ significantly (fig. 1). The serum level of PICP and the levels of PIIINP in serum, BALF and ELF did not show significant differences between any of these groups (table 2 ). PIIINP was detectable in BALF in $1 / 5$ patients with asbestosis, in $3 / 25$ patients with pleural involvement and in none of the patients without pleural involvement (data not shown). One patient of the asbestosis group had an elevated S-ALAT value, suggesting a possible hepatic lesion. As liver diseases are known to elevate S-PIIINP [19], the value of this patient was excluded.

The patients without parenchymal involvement were classified into three categories according to the number of asbestos bodies detectable in BALF. There were 12 patients with no $\mathrm{AB}, 14$ patients with $0-1 \mathrm{AB} \cdot \mathrm{mL}^{-1}$ and 11 patients with $>1 \mathrm{AB} \cdot \mathrm{mL}^{-1}$ in BALF. The levels of PICP and PIIINP in BALF did not differ significantly between the three groups (fig. 2).
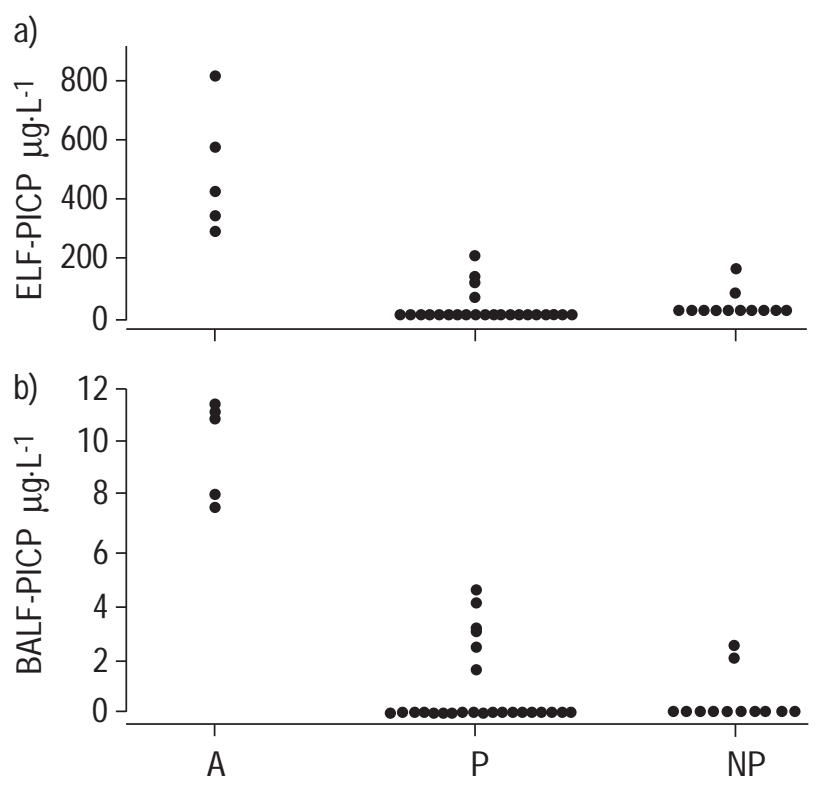

Fig. 1. - Individual values of carboxyterminal propeptide of type I procollagen (PICP) in a) epithelial lining fluid (ELF) and b) bronchoalveolar lavage fluid (BALF) in patients with asbestosis (A) $(n=5)$, pleural plaque disease $(\mathrm{P})(\mathrm{n}=25)$ and with no changes in chest radiographs $(\mathrm{NP})$ $(n=12)$. ELF-PICP not available in 1 patient in $P$ group because of missing BALF-Urea.

All the patients with exposure to asbestos fibres without parenchymal involvement were invited for a control visit after a follow-up period of 7 yrs. Three patients had died in conditions unrelated to asbestos; $30(81 \%)$ of the 37 patients responded. None of these patients had developed asbestosis when assessed with chest radiographs and diffusion capacity. One patient had an infiltrate caused by a lung carcinoma and 1 patient had a previously diagnosed pulmonary metastasis of a thyroid malignancy. The pleural plaques had progressed only in 1 patient. None of those with normal chest radiographs had developed plaques.

\section{Discussion}

This study shows that subjects with asbestosis have a higher level of the PICP in BALF and ELF compared to those exposed to asbestos fibres without parenchymal involvement. The PIIINP was detectable only in a few cases with or without asbestosis. The serum levels of PICP and PIIINP did not differ between the patients with asbestosis

Table 2. - Concentrations of PIIINP and PICP in serum, BALF, and ELF in patients with asbestosis and patients without parenchymal involvement

\begin{tabular}{|c|c|c|c|c|}
\hline & \multicolumn{2}{|c|}{ Asbestosis } & \multicolumn{2}{|c|}{ No parenchymal involvement } \\
\hline & Mean \pm SD & Median & Mean \pm SD & Median \\
\hline SPIIINP $^{\dagger} \mu \mathrm{g} \cdot \mathrm{L}^{-1}$ & $2.3 \pm 0.7$ & $2.5^{+}$ & $2.8 \pm 0.8$ & 2.7 \\
\hline $\mathrm{SPICP}^{\dagger} \mu \mathrm{g} \cdot \mathrm{L}^{-1}$ & $108.6 \pm 28.2$ & 104.5 & $120.4 \pm 33.7$ & 114.0 \\
\hline BALF-PIIINP $\mu \mathrm{g} \cdot \mathrm{L}^{-1}$ & $0.2 \pm 0.4$ & 0 & $0.1 \pm 0.6$ & 0 \\
\hline BALF-PICP $\mu \mathrm{g} \cdot \mathrm{L}^{-1}$ & $9.8 \pm 1.8$ & 10.8 & $0.6 \pm 1.3$ & $0 * * *$ \\
\hline ELF-PIIICP $\mu \mathrm{g} \cdot \mathrm{L}^{-1}$ & $11.6 \pm 25.9$ & 0 & $5.4^{\mathbf{s}} \pm 33.8$ & 0 \\
\hline ELF-PICP $\mu \mathrm{g} \cdot \mathrm{L}^{-1}$ & $488.9 \pm 208.8$ & 423.9 & $22.6^{\S} \pm 50.6$ & $0 * * *$ \\
\hline
\end{tabular}

***: $\mathrm{p}<0.001 .{ }^{\dagger}$ : available from 36 patients with no parenchymal involvement; $*: 1$ patient excluded because of elevated serum alanine aminotransferase; ${ }^{\S}$ : epithelial lining fluid (ELF) value not available in 1 patient with no parenchymal involvement because of missing bronchoalveolar lavage fluid (BALF)-Urea. S-PIIINP: serum aminoterminal propeptide of type III procollagen; S-PICP: serum carboxyterminal propeptide of type I procollagen. The significance of the difference was tested with the Mann-Whitney U-test. 

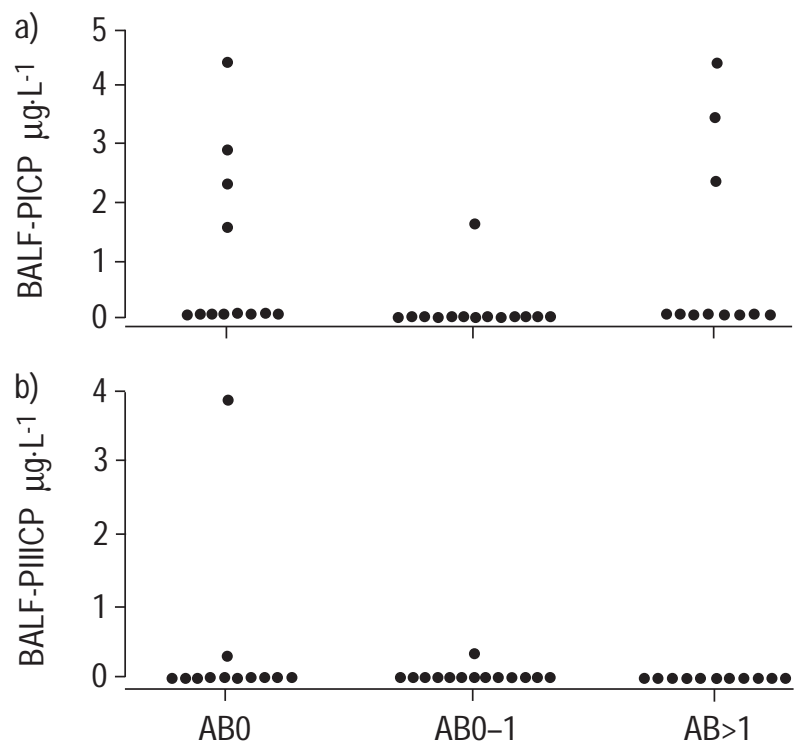

Fig. 2. - Individual values of a) carboxyterminal propeptide of type I procollagen (PICP) and $b$ ) aminoterminal propeptide of type III procollagen (PIIINP) in bronchoalveolar lavage fluid (BALF) from patients exposed to asbestos without parenchymal involvement compared with the number of asbestos bodies (AB) in BALF. $A B 0, n=12 ; A B 0-1$, $\mathrm{n}=14 ; \mathrm{AB}>1, \mathrm{n}=11$.

and those exposed to asbestos fibres without parenchymal involvement. None of the patients developed asbestosis during the follow-up period of 7 yrs.

PICP has not been previously investigated in the serum, BALF or ELF in pneumoconiosis. It has recently been observed that BALF-PICP is not detectable in patients with minor respiratory symptoms without pulmonary involvement [7]. Elevated PICP in BALF and ELF has also been detected in sarcoidosis $\left(3.7 \mu \mathrm{g} \cdot \mathrm{L}^{-1}\right.$ and $\left.290 \mu \mathrm{g} \cdot \mathrm{L}^{-1}\right)$ [7] and fibrosing alveolitis $\left(13.6 \mu \mathrm{g} \cdot \mathrm{L}^{-1}\right.$ and $\left.476.5 \mu \mathrm{g} \cdot \mathrm{L}^{-1}\right)$ [5]. In the present study all of the patients with asbestosis had elevated PICP in BALF and ELF. These values (11.6 $\mu \mathrm{g} \cdot \mathrm{L}^{-1}$ and $\left.488.9 \mu \mathrm{g} \cdot \mathrm{L}^{-1}\right)$ were very close to the findings made in fibrosing alveolitis, suggesting accumulation and active synthesis of type I collagen in the lungs of these patients. The levels of BALF- and ELF-PICP were low, but detectable in $8 / 37$ patients with exposure to asbestos fibres without parenchymal involvement. Detectable PICP in BALF did not correlate with either the presence of asbestos bodies in BALF or the prevalence of plaques. These results suggest that BALF- and ELF-PICP can be used in the evaluation of asbestos-related parenchymal disease, but they are not useful in the assessment of exposure to asbestos fibres. Since most of the PICP in serum is derived from bone, SPICP has no role in the assessment of pulmonary fibrosis [17]. In agreement with this, the level of PICP in the serum of the patients in the current study was similar to the PICP level observed in the serum samples of the control subjects in other recent studies $[5,7]$. Since the latency from the exposure to the onset of an asbestos-related pulmonary disease is long, the follow-up time of $7 \mathrm{yrs}$ does not exclude potential development of asbestosis in the 6 patients with elevated PICP in BALF and ELF and pleural plaques.

The PIIINP has been widely studied in BALF and serum of patients with sarcoidosis [4, 6, 7, 20-23]. Although many results have been contradictory, most studies suggest that elevated BALF-PIIINP is associated with disease activity. The level of PIIINP in BALF has also been shown to be elevated in fibrosing alveolitis, but the correlation of BALF-PIIINP with the activity of the disease and its role as a prognostic marker have, however, remained unclear [3-5, 24-26].

There are only a few studies on procollagen propeptides in pneumoconiosis, and most of them have been conducted on experimental animals. The exposures in most experimental models have included high and toxic concentrations of fibres. Exposure of rats to asbestos fibres for 2 weeks resulted in increased deposition of type III collagen and, later, type I collagen in the lungs [9]. Exposure of sheep to asbestos fibres also resulted in elevated levels of BALF-PIIINP after 60 days of follow-up [10], but no difference was found compared to the control values after 18 months [11]. Silica-exposed sheep showed no changes in the BALF-PIIINP levels after 9 months follow-up [27]. Experiments with rats exposed to silica showed that the messenger ribonucleic acid (mRNA) of type III procollagen increased earlier than type I procollagen when assessed by in situ hybridization [28].

The few studies available on humans exposed to asbestos fibres do not offer remarkable information about PIIINP in asbestos-related pulmonary diseases. SPIIINP appeared to be higher in the asbestos workers who developed radiologically interstitial fibrosis [13]. PIIINP in BALF was similar in the control subjects and in the asbestos workers without disease, but it was significantly elevated in the asbestos workers with asbestos-associated alveolitis and asbestosis [12]. Information on PIIINP in other types of pneumoconiosis is also limited. Silicaexposed workers had elevated PIIINP in BALF, but this change was only seen in progressive disease [29]. In coal workers' pneumoconiosis, SPIIINP was not a marker of interstitial or respiratory effects of coal dust [30]. Furthermore, SPIIINP did not predict the development of pneumoconiosis in coal workers during 5 yrs follow-up [31]. The role of SPIIINP as a biomarker of any pneumoconiosis has been considered unclear [32]. The present study included 5 patients with asbestosis and only one of them had a detectable PIIINP in BALF. Of the 37 patients exposed to asbestos fibres, but without asbestosis, three had detectable BALF-PIIINP. These values of PIIINP in BALF and serum were similar to those of a control group published recently [7]. The levels of PIIINP in BALF were similar regardless of the asbestos exposure estimated on the basis of the concentration of asbestos bodies in BALF or existing pleural plaques. In a previous study, the levels of PIIINP in BALF and ELF were higher in acute sarcoidosis than in fibrosing alveolitis $[5,7]$. The previous and present results suggest that low levels of PIIINP in BALF and ELF in parenchymal lung diseases, such as asbestosis, are probably related to low inflammatory activity in the lungs, and that PIIINP cannot be used in the assessment of asbestosis or asbestos-related pleural disease.

In conclusion, the carboxyterminal propeptidase of type I procollagen was highly elevated in the bronchoalveolar lavage fluid and epithelial lining fluid of the patients with asbestosis, suggesting that carboxyterminal propeptidase of type I procollagen could be used as a marker of collagen metabolism in the assessment of 
parenchymal involvement in individuals exposed to asbestos fibres. The unchanged levels of aminoterminal propeptide of type III procollagen in bronchoalveolar lavage fluid, epithelial lining fluid and serum suggest low inflammatory activity in the lungs with asbestosis, and indicate that aminoterminal propeptide of type III procollagen has no role in the assessment of asbestosis or asbestos related plaque disease in humans.

Acknowledgements. The authors wish to thank R. Jokela, J. Träskelin and T. Ahonen for their expert technical assistance.

\section{References}

1. Bateman ED, Turner-Warwick M, Adelman-Grill BC. Immunohistochemical study of collagen types in human foetal lung and fibrotic lung disease. Thorax 1981; 36: 645-653.

2. Prockop D, Kivirikko K, Tuderman L, Guzman N. The biosynthesis of collagen and its disorders [Review]. $N$ Engl J Med 1979; 301: 13-23.

3. Bjermer L, Lundgren R, Hällgren R. Hyaluronan and type III procollagen peptide concentrations in bronchoalveolar lavage fluid in idiopathic pulmonary fibrosis. Thorax 1989; 44: 126-131.

4. Low RB, Cutroneo KR, Davis GS, Giancola MS. Lavage type III procollagen N-terminal peptides in human pulmonary fibrosis and sarcoidosis. Lab Invest 1983; 48: 755-759.

5. Lammi L, Ryhänen L, Lakari E, et al. Type III and Type I procollagen markers in fibrosing alveolitis. Am J Respir Dis Crit Care Med 1999; 159: 818-823.

6. Bjermer L, Thunell M, Hällgren R. Procollagen III peptide in bronchoalveolar lavage fluid. A potential marker of altered collagen synthesis reflecting pulmonary disease in sarcoidosis. Lab Invest 1986; 55: 654-656.

7. Lammi L, Kinnula V, Lähde S, et al. Propeptide levels of type III and type I procollagen in the serum and bronchoalveolar lavage fluid of patients with pulmonary sarcoidosis. Eur Respir J 1997; 10: 2725-2730.

8. Risteli J, Risteli L. Analysing connective tissue metabolites in human serum. Biochemical, physiological and methodological aspects. J Hepat 1995; 22 (Suppl. 2): 77-81.

9. Arden M, Adamson I. Collagen synthesis and degradation during the development of asbestos induced pulmonary fibrosis. Exp Lung Res 1992; 18: 9-20.

10. Bégin R, Masse S, Rola-Pleszczynski M, Boctor M, Drapeau G. Asbestos exposure dose - bronchoalveolar milieu response in asbestos workers and the sheep model: evidences of a threshold for chrysotile-induced fibrosis. Drug Chem Toxicol 1987; 10: 87-107.

11. Bégin R, Cantin A, Sebastian P. Chrysotile asbestos exposures can produce an alveolitis with limited fibrosing activity in a subset of high retainer sheep. Eur Respir $J$ 1990; 3: 81-90.

12. Bégin R, Martel B, Desmarais Y, et al. Fibronectin and procollagen 3 levels in bronchoalveolar lavage of asbestos-exposed human subjects and sheep. Chest 1986; 89: 237-243.

13. Cavalleri A, Gobba F, Bacchella L, Luberto F, Ziccardi A. Serum type III procollagen peptide in asbestos workers: an early indicator of pulmonary fibrosis. $\mathrm{Br} J$ Ind Med 1988; 45: 818-823.

14. Davis GS. Bronchoalveolar lavage in interstitial lung disease. Semin Respir Crit Care Med 1994; 15: 37-60.
15. Churg A, Warnock M. Asbestos and other ferruginous bodies: their formation and clinical significance. $\mathrm{Am} \mathrm{J}$ Pathol 1981; 102: 447-456.

16. Risteli J, Niemi S, Trivedi P, Mowat AP, Risteli L. Rapid equilibrium radioimmunoassay for the amino-terminal propeptide human type III procollagen. Clin Chem 1988; 34: 715-718.

17. Melkko J, Niemi S, Risteli L, Risteli J. Radioimmunoassay of the carboxyterminal propeptide of human type I procollagen. Clin Chem 1990; 36: 1328-1332.

18. Rennard S, Basset G, Lecossier D, et al. Estimation of absolute volume of epithelial lining fluid recovered by bronchoalveolar lavage by using urea as an endogenous marker of dilution. J Appl Physiol 1986; 60: 532-538.

19. Raedsch R, Stiehl A, Waldherr R, et al. Procollagen-typeIII-peptide serum concentrations in chronic persistent and chronic active hepatitis and in cirrhosis of the liver and their diagnostic value. Zeitschr Gastroenterol 1982; 20: 738-743.

20. Pohl WR, Thompson AB, Köhn H, et al. Serum procollagen III peptide levels in subjects with sarcoidosis. A 5-year follow-up study. Am Rev Respir Dis 1992; 145: 412-417.

21. Luisetti M, Bulgheroni A, Baccella L, Pasturenzi L, Aprile C. Elevated serum procollagen III aminopeptide levels in sarcoidosis. Chest 1990; 98: 1414-1420.

22. Milman N, Kristensen MS, Bentsen K, Grode G, Fredriksen J. Hyaluronan and procollagen type III aminoterminal peptide in serum and bronchoalveolar lavage fluid in patients with pulmonary sarcoidosis. Sarcoidosis 1995; 12: $38-41$.

23. O'Connor C, Ward K, van Breda A, Mcllgorm A, FitzGerald MX. Type 3 procollagen peptide in bronchoalveolar lavage fluid: poor indicator of course and prognosis in sarcoidosis. Chest 1989; 96: 339-344.

24. Cantin A, Boileau R, Begin R. Increased procollagen III aminoterminal peptide-related antigens and fibroblast growth signals in the lungs of patients with idiopathic pulmonary fibrosis. Am Rev Respir Dis 1988; 137: 572-578.

25. Harrison NK, McAnulty RJ, Kimpton WG, Fraser JRE, Laurent TC, Laurent GJ. Heterogeneity of type III procollagen N-terminal peptides in BAL fluid from normal and fibrotic lungs. Eur Respir J 1993; 6: 1443-1448.

26. Low RB, Giancola MS, King TE, Chapitis J, Vacek P, Davis GS. Serum and bronchoalveolar lavage of N-terminal type III procollagen peptides in idiopathic pulmonary fibrosis. Am Rev Respir Dis 1992; 146: 701-706.

27. Bégin R, Dufresne A, Cantin A, Possmayer F, Sebastien P. Quartz exposure, retention and early silicosis in sheep. Exp Lung Res 1989; 15: 409-428.

28. Vuorio E, Mäkelä J, Vuorio T, Poole A, Wagner J. Characterization of excessive collagen production during development of pulmonary fibrosis induced by chronic silica inhalation in rats. Br J Exp Pathol 1989; 70: 305-315.

29. Bégin R, Cantin A, Boileau R, Bisson G. Spectrum of alveolitis in quartz-exposed human subjects. Chest 1987; 92: 1061-1067.

30. Schins R, Lamers R, Preat B, Borm P. Evaluation of serum type III procollagen peptide as an exposure marker in retired coal workers. Int Arc Occup Environ Health 1995; 66: 413-419.

31. Schins R, Borm P. Serum procollagen type III peptide in coal workers' pneumoconiosis: a five year follow up study. Exp Lung Res 1994; 20: 445-455.

32. Borm P. Biological markers and occupational lung disease: mineral dust-induced respiratory disorders. Exp Lung Res 1994; 20: 457-470. 